\title{
Brief Analysis on the Current Situation and Problems of Brand Advertisement in China's Internet Financial Industry
}

\author{
En Deng ${ }^{1,2}$, Yuanji Huang ${ }^{3,4^{*}}$ \\ ${ }^{1}$ College of Economics and Trade, Hunan University, Changsha 410082, China; \\ ${ }^{2}$ Hunan Radio and Television University, Changsha 410004, China; \\ ${ }^{3}$ Hunan Provincial Social Science Association, Changsha 410003, China; \\ ${ }^{4}$ Hunan Agricultural University, Changsha 410128, China. \\ *The corresponding author
}

\begin{abstract}
In recent years, Internet thinking is like a Renaissance of contemporary Chinese financial industry. It is profoundly changing the pattern and development of traditional financial industry. In the traditional industry, the formation and dissemination of a brand sometimes need dozens or even hundreds of years for accumulation and promotion, and in today, some emerging Internet financial brand from birth to a household name, just needing ten months' time. It can be seen that brand communication is essential for the development of Internet financial enterprises, and is the most critical part impacting consumer brand selection behavior. Based on the development reality of China's Internet financial industry, this paper chooses the brand communication perspective to study the present situation of the development of Internet financial industry brand, and analyzes the problems of brand communication as the basis for the brand communication and provide analysis reference for the actual business managers.
\end{abstract}

Keywords: Internet financial industry; Brand communication; Status; Problem

\section{浅析中国互联网金融业品牌传播的现状与问题}

\author{
邓恩 ${ }^{1,2}$ ，黄渊基 ${ }^{3,4 *}$ ，
}

（1. 湖南大学经济与贸易学院，湖南长沙 410082；2. 湖南广播电视大学，湖南长沙 410004

3.湖南省社科联 湖南长沙 410003；4. 湖南农业大学 湖南长沙 410128)

摘要：近年来，互联网思维如同一场类似于中国当代金融业的文艺复兴，正深刻地改变着传统金融业的格局和业态发 展。在传统行业, 一个品牌的形成和传播, 有时需要几十甚至上百年的积累和推广, 而在当今, 一些新兴互联网金融品牌从 诞生到家喻户晓，也就是短短十几个月的时间。可见，品牌传播对于互联网金融企业的发展至关重要，是影响消费者品牌选 择行为的最关键一环。该文从中国互联网金融业的发展现实出发, 选取品牌传播视角来研究当前互联网金融业品牌传播的发 展现状，并以此为基础来分析其品牌传播存在的问题，为品牌传播的研究者和实际业务管理者提供分析参考。

关键词：互联网金融业；品牌传播；现状；问题

中图分类号: F49.1 文献标志码: A

引言

通常来说，互联网金融企业的品牌核心竞争力就是通过各类互联网金融业务开展过程中所展示出的凝 聚力与影响力, 即其他竞争对手难以超越、替代的被市场或客户认同的企业形象、信誉、内在品牌价值等。 显然, 品牌传播对于互联网金融企业的发展至关重要, 是影响消费者品牌选择行为的最关键一环。然而, 总体上看, 中国互联网金融公司通过品牌传播运营来影响消费者的品牌选择行为仍处于初级阶段, 迫切需 要学习借鉴先进经验, 如何通过提升互联网金融品牌传播效果来引导消费者的品牌选择行为, 这已经引起 
了业界、政府、学者的广泛关注。但我国互联网金融产业发展时间不长、品牌传播效果差异较大, 成功典 型不多，互联网金融业品牌传播的发展现状到底如何? 从品牌传播视角来看，还存在哪些问题? 这些问题 成为本文选题、研究的起点和目标。

\section{1. 互联网金融业品牌传播现状}

自从中国国内的互联网金融产品开始出现时，有关互联网金融品牌建设的实践探索就已经开始出现。 如 “支付宝” 品牌两个著名的品牌宣传片的策划与投放、工行互联网金融品牌 “ $\mathrm{e}-\mathrm{ICBC}$ ” 的高调发布、宜 信开启的 “敢 2” 品牌营销活动, 等等。这些互联网金融公司都是通过各类机会来塑造互联网金融品牌, 并通过有效的传播策略来提升品牌知名度和美誉度。现阶段我国互联网金融品牌传播的现状有以下几个方 面:

\section{1 信用、便捷、稳健、创新的理念成为互联网金融品牌传播的重要内容}

2000 年以来，我国互联网金融品牌呈现出雨后春笋般爆发式发展，各个子行业分布形成了各自独特的 品牌形象和个性，其中以第三方支付、P2P、互联网保险、互联网理财、传统金融机构互联网平台的发展 最具代表性。支付宝借助品牌形象宣传片《郑棒棒的故事》、《爱心联盟》, 将 “信任与托付”、“便捷与稳 健” 作为支付宝用户共同拥有、彼此认同的理念, 进行品牌塑造和传播, 迅速提升了支付宝的品牌知名度 和美誉度。品牌的作用在财富管理行业常常易被忽略, 同行业内的金融机构往往提供类似的产品, 品牌之 间的边界和差异趋向模糊。财富管理不是单纯获得收益, 取得财富的增长, 而是委托专业机构进行专业管 理, 实现客户自由、高效、专业的理财需求和理想。在 2012 年宜信提出了 “财富管理好, 幸福自然来” 的品牌运营理念, 并运用旗舰店的方式来传播和推广, 使消费者能随时随地地感受和触摸到该品牌, 在消 费者心目中形成全面、立体、丰满的品牌形象。PayPal 是 eBay 旗下的一家公司, 成立于 1998 年, 主要为 大众提供在线安全、便捷、简单的收付款服务。PayPal 所从事的所有事务都秉承以人为本的原则。2014 年, PayPal 推出该公司历史上首个全球品牌宣传计划, 诠释该公司引领和倡导的独特资金使用理念: 让人 们可以随时随地以一种更轻松、更安全、更便于操作的方式进行支付。新宣传计划除营销之外, 还采用新 的品牌和最新的 PayPal 标识, 新标识采用更加现代和注重移动体验的外观。新的 Logo 用两个颜色深浅不 一的斜体 “P” 当作主要形象, 主要体现了 “乐观、进步、充满能量” 的经营理念。此外, 与传统金融相 比, 互联网金融的网络交易模式, 更需要 “信用与稳健”, 这也成为品牌传播的重要内容, 当然也有一些 互联网金融品牌以高收益、低风险保障等来作为品牌传播的主要要素。

\section{2 网络媒体、社交媒体、户外广告成为互联网金融业品牌的主要传播媒介}

与传统金融机构的实体营业厅不同，互联网金融的产品和服务都是依赖于网络平台，因此消费者基本 上首先应该是一个网民，能够熟练使用台式电脑、平板电脑或手机终端上网。将互联网金融业品牌传播主 阵地确立为网络媒体和社交媒体上也就不足为奇了。如今我们随意打开国内最受欢迎十大门户网站, 都可 以任意在主要界面找到金融栏目，其中的二级栏目中都存在互联网金融的品牌网站链接或品牌传播推广的 浮动广告浮窗, 如搜狐理财频道下有互联网金融子频道、风凰网有自己的著名互联网金融产品---凤凰理 财、新华网在主页上有互联网金融平台 “一路财富” 的品牌浮窗广告 “年化 $10.8 \%$ 给你稳定的小时代” 及 链接网站、在财经栏目下就是自己的互联网理财产品---新华・理财、新财汇栏目等等。Paypal 也于 2014 年首次推出了全球性广告传播宣传, 由 Havas Worldwide 提出创意, 并通过电视、平面、互动、户外、店 
面等多种形式在在线渠道上上线运营，包括 PayPal. com、PayPa1-Forward. com 和社交媒体渠道进行传播 推广。全球各种各样的户外广告中, 当属世界经济中心一纽约曼哈顿时代广场为最, 广场周边附近聚集着 全球顶尖品牌的形象广告, 其中的纳斯达克大屏幕则成为无数世界顶尖品牌的兵家必争之地。2015 年新年 伊始，纳斯达克旗下最大的电子屏幕上，出现了神秘的东方文字---来自中国的互联网金融平台 “理财屋 (licaiwu. com)”。理财屋互联网金融平台已于 2015 年 1 月 1 日零点上线, 仅 30 分钟内, 理财屋平台春节 宝一期产品便一抢而空。此番对外展示, 是理财屋平台借上线之际, 试图让全世界看到中国最权威的全抵 押互联网金融平台--理财屋的优秀运营模式, 并吸引全球投资者的目光, 也是一次最为成功的品牌传播和 展示。显然, 在互联网金融业和互联网迅猛发展的今天, 网络媒体、社交媒体已经代替电视、广播等传统 传播媒介成为互联网金融业品牌传播的主战场。

\section{3 品牌塑造和传播受到互联网金融业企业的普遍重视}

伴随互联网金融的迅猛发展，互联网金融品牌日益受到重视。很多今天还是新产品名称，甚至只是一 个普通商标, 但在网络时代很可能在不久的将来, 成为一个著名商标和知名品牌。例如阿里巴巴集团早在 2013 年 6 月即向商标局提交了余额宝商标注册申请, 从颁发注册证那天起, 余额宝就是一件响当当的商 标。自从阿里巴巴的余额宝成为家喻户晓的著名品牌后, 互联网上新产品频出, 零钱宝、收益宝、活期宝 等各类 “宝宝” 产品以及传统电商易信、苏宁、京东、360 等也加入互联网金融行业，推出各自产品，一 时间, 产品战慢慢变成了品牌战。各类互联网金融企业纷纷砸重金塑造自己的新品牌, 打造有个性和有影 响力的品牌。成果也是显著的：2014 中国互联网金融大会春季峰会上揭晓了 “中国互联网金融百强品牌” 奖, 就是表彰了一批互联网金融年度行业的典型代表企业, 目的在于增强互联网金融行业和企业的品牌建 设。峰会上出现了 P 2P、众筹、银行、基金、证券、第三方支付等众多互联网金融的细分领域。品牌传播 也是国际互联网金融巨头最为重视的头等大事, 如针对外界越来越多的对 PayPal 的负面评论, PayPal 公 司总裁 Davia Marcus 为了挽回 PayPa1 在公众心目中的品牌形象, 甚至亲自给用户发送致歉信并且承诺将 快速调整公司的状况。P2P 行业的鼻祖、即将登陆美国纳斯达克市场的准上市公司----美国 Lending Club, 就宣称公司进行 IP0 的主要目的是为了扩大品牌知名度和影响力。传统金融机构也快速发力, 着力打造网 络链金融品牌, 例如北京银行的 “网络链”, 即是一款网络供应链品牌, 其与第三方支付公司快钱签署了 全面战略合作协议。有“宇宙第一大行”之称的中国工商银行在 2015 年春季发布了其精心打造的“ $\mathrm{e}$ - ICBC” 品牌, 高调进军互联网金融业。互联网金融品牌已经日益深化为从业者、消费者、投资者等人群的一种标 志识别的感官认知, 品牌代表着产品质量和服务水平, 我国的互联网金融品牌传播也伴随着行业的发展而 不断走向成熟, 并日益被各界所重视。

\section{2 互联网金融业品牌传播存在的问题}

互联网金融业品牌的传播和推广是一个复杂而系统的工程，需要各个互联网金融企业有清晰的传播战 略、目标和有效的实施方案、并持之以恒地不断坚持和完善, 才能把塑造的品牌逐步建立起来, 形成品牌 的美誉度和知名度。我国互联网金融业发展时间虽然不长, 却取得了非常不错的成果, 像支付宝等品牌已 经深入人心，家喻户晓，但也存在一些突出问题，主要有以下几方面:

\section{1 互联网金融企业对品牌建设和传播认识不清晰、重视不够}

2010 年以来, 互联网金融行业发展迅猛, 甚至被称为野蛮生长。特别是 P2P 网贷平台尤其引人关注, 
大量平台在不断涌现的同时，平台倒闭、老板跑路的消息也此起彼伏。这说明市场竞争激烈、不少互联网 金融品牌还缺乏品牌塑造和维护意识，以致金融信用、企业品牌声誉都缺失，遇到资金断裂等金融风险等 问题时, 就容易放弃品牌甚至平台。其实对于互联网金融行业而言, 绝大部分业务都在网上开展, 品牌传 播和信誉尤为重要, 平台品牌之间的竞争最关键就是要重视在消费者中塑造良好的品牌和声誉, 这也是互 联网金融业企业的品牌传播生命线与核心竞争力所在。

中国互联网金融的各个子行业还处于初创时期，市场上比较突出的品牌还较少，互联网金融企业中大 多数只是打算塑造品牌来推动销售，比如采用各种网络渠道来对抗国外互联网金融机构如 Lending Club 和 Prosper 等, 虽然品牌能做到短期内让人家喻户晓, 但缺乏对品牌建设和传播的认识不清晰或者重视不 够, 则大多数企业对整个行业而言最终只是在互联网金融领域中增加了一家毫无品牌特色的平台。反之, 则有可能在互联网金融行业引入一家变革式企业。

\section{2 缺乏明确、长远传播目标}

现阶段中国的互联网金融品牌传播和推广中，很多时候更为看重眼前利益，平台之间以价格战为主要 竞争手段, 上级对下级部门和员工的考核以业务数量为主, 眼光不够长远。这如同上个世纪 90 年代末, 中国国内很多人看重电子商务中的网上书店，因为市场从无到有，竞争很不充分，不少人淘到了金，挣到 了原始积累。可是, 很快由于美国电子商务巨头亚马逊进入中国市场, 制定并实施了明确的品牌传播目标, 鲜明而成功的品牌形象得以迅速确立和成功塑造, 随后与国内的品牌成功塑造者---当当网一道垄断了整 个网上图书销售市场, 其他中小企业也就只有退出或转行的唯一两个选项了。一个证据就是, 今天没有一 个商家会想着去网上卖书, 因为市场已经难以渗透进去。历史有惊人的相似性。今天的互联网金融企业应 当尽快打造自己的品牌传播战略和明确的长远目标, 明确传播的定位和目标受众。在宏观上与企业的定位 一致, 在微观上与阶段性目标和主体客户一致。通过细分市场来强化品牌传播的目标性和针对性。只有清 醒认识并重视品牌传播的长远作用, 制定和实施明确有效的品牌传播目标和长远计划, 在品牌传播和推广 最终开花结果时, 也就能体现出其巨大的潜在价值, 企业品牌生命之树也就能长青。

\section{3 缺乏品牌传播模式与战略规划}

对于互联网金融业品牌传播，很多企业还只是停留在设计精美的品牌标识、建设功能强大、美观大方 的平台网站、征集品牌宣传口号、制作几个品牌宣传片上, 没有上升到传播模式的研究和战略高度上来。 品牌宣传工作缺乏系统规划和传播模式, 必然出现难以从一而终。一些互联网金融企业从开始传播品牌的 那一刻起, 就开始高调宣传品牌, 召集品牌宣传精英, 不断通过各类活动宣传造势, 宣扬企业文化、品牌 形象。市场上什么品牌宣传方式最热、什么最火, 就喊什么口号, 打什么牌, 品牌塑造不可谓不强势、品 牌诠释不可谓不到位。可是, 由于缺乏传播模式和战略规划, 先天体系不足, 往往雷声大雨点小, 一场声 势浩大的品牌传播运动过后, 不用多久就无疾而终、销声匿迹的不在少数。可见, 一个普遍的问题是, 我 国互联网金融企业大多数没有从战略规划角度建立自己的品牌传播模式。纵观国内外其他行业的发展历 程, 成功的品牌无一例外地都是依赖于一套有战略规划、系统完整、适合企业自身和市场需求的品牌传播 模式。互联网金融行业的发展和竞争日益激烈，靠运气、投机取巧，难以长久，企业间品牌的竞争说到底 最终是靠品牌模式和战略体系的比拼。探索出自己的品牌传播模式、做好品牌战略规划, 建立完善的品牌 传播体系，是系统而复杂的战略性工程，但也只有这样，在互联网金融市场上才能立于不败之地。 


\section{4 传播推广手段有待创新}

品牌传播和战略规划的实施必然通过传播手段---媒介来实现。我国互联网金融品牌传播大多以互联 网为主要媒介, 这符合品牌传播受众主要为网民的特征, 但也存在传播媒介和手段单一、传播形式缺乏创 新的问题。随着社交媒体在移动互联网终端的迅速运用, 传统的品牌传播模式对提升形象、塑造品牌的作 用正在下降, 这对互联网金融企业提出了品牌传播管理模式变革的要求。很多企业还没有跟上变革的步伐, 传统的广告、电视等媒体作为传播手段, 加上缺乏新意的内容设计, 品牌传播效果必然收效甚微。当然, 也有部分互联网金融企业已经认识到这一点, 如支付宝等企业综合利用社交网站、微博、微信朋友圈、公 众号、博客、论坛、播客、领英、视频网站等来形成综合立体的品牌传播平台, 创造新颖多样的内容设计, 以实现促进教育受众、推送品牌信息、增进了解的目的。传播效果则是类似病毒传播的方式, 迅速将企业 品牌、产品功能、企业活动甚至企业危机事件转化成正面信息快速传播发散出去, 把数字媒体渠道作为重 点领域来打造口碑和提升品牌传播效果。

\section{3 结语}

该文结合应用经济学与媒介经济学的相关理论，选择品牌传播的视角，在对中国互联网金融业品牌传 播发展现状分析的基础上，从互联网金融企业对品牌建设和传播认识不够、缺乏明确和长远传播目标、缺 乏品牌传播模式与战略规划、传播推广手段有待创新等四个方面提出了存在的问题。试图为互联网金融业 企业提供从品牌传播入手来提高品牌传播效果, 从而引导消费者品牌选择行为提供制定决策的参考和研究 基础，为促进我国互联网金融业健康快速发展提供现状研究支撑。

\section{致谢}

本文系国家社科基金“大学生网络素养与社会主义核心价值观认同研究”（16CKS045）阶段性成果。

\section{Acknowledgement}

This article is stage result of " Research on college students network literacy and socialism core values recognition" supported by the National Social Science Fund (16CKS045) .

\section{参考文献:}

[1] 在线支付服务公司 PayPal 启用新 LOGO http://www. logonews. cn/paypal-new-logo-2014. html

[2] 理财屋强势登陆纳斯达克 新年进军国际市场 2015 年 01 月 13 日 09: 15 来源：中国网 http://finance. eastmoney. com/news/1354, 20150113467090836. html

[3] 互联网金融商标暗战 2014-03-06 整理编辑: 中国商标注册查询网

[4] 2014 中国互联网金融大会春季峰会盛大召开 中国网 www. china. com. cn 2014-04-02http: //finance. china. com. cn/ roll /20140402/2306917. shtml

[5］记者 间瑾. 全球 P2P 行业鼻祖上市在即 [N]．北京商报, 2014-12-05007.

[6] 杜玢．植根齐鲁大地 铸就优质品牌[N]．金融时报，2014-09-23005.

[7］潘玉．依托业内首个互联网金融品牌 “e-ICBC” 辽宁工行加速拥抱 “互联网+” [J]．辽宁经济, 2015, 07:90-91.

[8] 唐春燕. 关键事件营销描述方式对消费者品牌形象评价的影响研究 [D].江西师范大学, 2009.

[9] 史春魁. 金融风险管理理论论述 [J]．和田师范专科学校学报, 2007, 03:30-31.

[10］张勇. 我国企业信用管理现状及对策研究[硕士].江苏大学硕士学学位论文, 2007: 8.

作者简介: 邓恩 (1978--), 男, 湖南大学在读博士、湖南广播电视大学经济学副教授, 研究方向: 互联网金融、金融 经济学; 通信作者: 黄洲基（1980--）男, 湖南农业大学在读博士, 湖南省社科联副研究员, 研究方向: 金融经济。 


\section{References:}

[1] Online Payment Service Company PayPal Enable New LOGO http://www.logonews. cn/ paypal -new-logo-2014.html

[2] Financial home strongly landing Nasdaq, entering the international market in a new year on January 13, 2015 09:15 Source: China Network http://finance.eastmoney.com/news /1354, 20150113467090836.html

[3] Internet Financial Trademark Underground Race 2014-03-06 Arrange and Edit: Chinese trademark registration query network

[4] 2014 China Internet Finance Conference Spring Summit was held grandly, China Network www.china.com.cn 2014-04-02http://finance.china.com.cn/roll/20140402/2306917.shtml

[5] Reporter Yan Jin. Global P2P Industry Originator Listed Soon [N]. Beijing Daily, 2014-12-05007.

[6] Du Bin. Planted Qilu, Making High-Quality Brand [N]. Financial Times, 2014-09-23005.

[7] Pan Yu. Relying on the Industry's First Internet Financial Brand "e-ICBC" Liaoning ICBC Speed Up the Embrace of "Internet +" [Liaoning], Liaoning Economy, 2015, 07: 90-91.

[8] Tang Chunyan. Study on the Effect of Key Events Marketing Description for Consumer Brand Image Evaluation [D]. Jiangxi Normal University, 2009.

[9] Shi Chunkui. Theoretical Discussion of Financial Risk Management [J]. Journal of Hotan Teachers College, 2007, 03: 30-31.

[10]Zhang Yong. China's Enterprise Credit Management Status and Countermeasures [Master]. Jiangsu University Master's Degree thesis, 2007: 8. 